\title{
Male Behaviour Standards in Female Perception
}

\author{
Elena Maksimyuk, and Elena Tsoupikova \\ Siberian Automobile and Highway University, Mira Ave, 5, Omsk, Russia
}

\begin{abstract}
The paper is devoted to the analysis of male behaviour standards in female perception. Speech fragments taken from female dialogs explicate the presence of male behaviour standards in female consciousness. Cognitive and semantic analysis of female speech behavior peculiarities allowed us to reconstruct and describe female perception determined by social and cultural reality of linguistic and cultural society they live in. It was discovered that female perception was determined by social and cultural stereotypes and roles. This determination is realized in a strong need to correspond to these stereotypes and roles. Special attention is paid to the pragmatic functions of linguistic units, emotional attitude expressed in speech, to the types of communicative determination, to the evaluation scale and to the perception channel revealed in the process of communication.
\end{abstract}

\section{Introduction}

Linguistic works of recent years combine sociolinguistic, cultural and cognitive components, with the speaker in the centre of attention. One of the main functions of the language, in addition to the transmission of information and determination, is orientation of the person in his own cognitive space [1]. Language is considered as a system of orienting, where the circumstances play a decisive role [2].

Genderology is a relatively new branch of linguistics, but recently it has been actively developed. Genderology refers to the structures of language, recognizing their significant role in the mechanisms of cultural gender representation, which makes linguistics one of the most important scientific disciplines through which the process of knowledge of gender can be realized. Almost all areas of language can be studied in a gender aspect [3].

Gender studies have a sociocultural character. Intergender relations are actively manifested in the language. Gender is part of both collective and individual consciousness. The gender predetermines many physiological reactions, including speech. "Gender should be considered as a cognitive phenomenon, manifested in stereotypes fixed by the language" [4].

\section{Theory}

\subsection{Modern approaches}

In modern science, gender is interpreted not as a biological phenomenon, but as a multicomponent sociocultural phenomenon, it is explained by general tendency towards anthropocentrism and by the integration of various branches of humanitarian knowledge [5]. Gender is considered as a sociocultural construct, as a conventional phenomenon and as a discursive factor of variable intensity [6].

A significant achievement in the cognitive paradigm is the creation of the theory of the personality, its structure includes three levels: the linguistic picture of the world, the conceptual picture of the world and motivational and pragmatic attitudes [7].

\subsection{Object peculiarities}

The study of the emotional sphere of individuals has its own characteristics, because emotions are a part of intrapsychic phenomenon. "External manifestations of emotions inevitably has a partial fixation of several levels of linguistic personality, because cognitive, emotional and volitional processes are not isolated in the integral psychological structure of the personality, but it is functioning in unity, highlighting those that play the greater role in reflecting the world and regulating behaviour on this segment of human activity "[8]. Speech is one of the most universal and common ways of actualizing emotions. It is noteworthy that the language is dominated by the emotions of disapproval, i.e. negative emotions can be expressed much more diversely than positive ones [9]. Neutral means of language, depending on the author's intention, can become expressive [10]. There is an emotional, figurative, aesthetic transformation of the pragmatic meanings of linguistic units. All the knowledge about emotions the individual absorbs in the process of socialization. It is socialization that promotes that individual and situational experiences are constituted as social and typical experiences [10]. As social

* Corresponding author: Grechko_EV@mail.ru 
phenomena, emotions are subject to social regulation and control [10]. These rules are determined by the value scale of the society and they change in accordance with national history and culture. The study of the manifestation of gender characteristics allows us to reconstruct and describe the mental space mediated and determined by the social and cultural being of a particular cultural community.

\section{Task assignment}

\subsection{Aims}

This gender study is to reveal roles, norms, values, and personal traits [11] through the analysis of the pragmatic and communicative context in which gender is constructed, and to reveal the impact of extra- and intralinguistic factors on this process [12].

\subsection{Research focus}

Gender is a tool through which both individual and collective consciousness perceives and evaluates the world in the process of historical and cultural development [13]. The following factors of gender communicative behaviour were taken into consideration: sociability, emotionality, communicative affability, etiquette, control, communicative distance, the ratio of verbal and nonverbal components, and much more [14].

\section{Research data and methods}

\subsection{Data choice}

The discourse of the comedy "Deffchonki" is taken because behind the comedy often social and psychological, personal and public top problems are hidden. We tend to talk only about what is important for us. Cinematography is aimed at a mass addressee, focused on the core of social qualities and on what is perceived as a deviation from it. The study of the comedy allowed us to obtain information about the features of perception of reality, and its gender peculiarities.

Special attention was paid to the first episode, because it has the function of involving and attracting and keeping the audience attention.

\subsection{Data characteristics}

The comedy "Deffchonki" was chosen because the main theme of communication in it is everyday issues. In everyday communication, we often encounter nonstandard communicative situations, it is minimally conventionalized in comparison with other types of communication, but much richer in thematic content, in terms of organization and implementation, since the ethical and cultural filter is absent in most cases, the degree of freedom of the speaker is limited only by the presence of children or old people. Their presence automatically activates the action of these filters imposes many thematic, lexical, stylistic and even intonational taboos on speech.

The analysis of speech behaviour was made with the support on the components of the communicative situation (addressee, addressee, their social roles and intentions, subject of speech, etc.), which determine the form of the speech. The main method used in the research is cognitive and semantic analysis.

\section{Research analysis}

\subsection{Value hierarchy and receptive stereotypes}

During the analysis of speech discourse, it was noted that women often "invade" in the cognitive space of men:

- they build a value hierarchy for them:

(Zoya) Are you sick? It is already like his relative (about his attitude to the bottle of expensive alcohol). I saw how he grabbed it. He even gave it a name.

- they give the receptive stereotypes:

(Masha) Underwear is very important in a relationship. White is the colour of innocence, and you would remove the ambiguity of the situation. Red highlights the passion! But put on black. Black makes you slim!

- they play the role of a judge and approve the boundaries of what is permissible:

(accountant) He has the habit of taking the beauties to the most responsible positions.

Often, women make conclusions instead of man and then try to impose it on a man, this later leads to the conflict:

- plan for a man:

(Masha) Since childhood I've dreamed of two children - a girl and a boy. The main thing is to give birth before you are 26, and the second time before 28 years, so that the difference would be small, and the elder child could take the younger child to the kindergarten.

\subsection{Functions, emotional background, type of determination, values, scale, leading channel of perception, intensifiers}

The main function is to extend the cognitive program of the future to the partner, showing its importance and constructing a hierarchical system. Significance is transmitted through the image of the duration expressed by the lexeme in the phase meaning (since childhood). The duration of the repetition of the actions is laid down in predicative meanings (dreamed, take to the kindergarten). The hierarchy is built by consistent argumentation. Psycho emotional intensification is in the absolute beginning of the statement (the main thing). Then a complex syntactic structure is used to model the future.

Emotional background: desire.

The main type of determination: time (since childhood, before 26, before 28 years) and time-type 
determinative constructions (elder, younger), explicate the desired temporary milestones of future events. The more accurately the program matches the reality, the happier the woman will be (in her opinion). The goal is to realize oneself in the social roles of wife and mother.

Values: social roles of wife and mother.

Scale: the realization of oneself in the social roles of wife and mother for the woman is evaluated as "good"; non-realization is evaluated as "bad" for the future.

The leading channel of perception: visual - images ( $a$ girl, a boy, to the kindergarten).

The goal is to motivate the interlocutor to join this program of the future.

Intensifiers:

Morphological: suffixes (elder, younger).

Lexical: dreamed, main thing.

Syntactical: transmitting future plans and time distribution (and, to, and).

At the same time, a woman does not notice that this is her plans, her dream and her future program. The action is produced by her, explicates her internal cognitive space, existing separately from the male one, so it is not surprising that it goes against his cognitive program of the future. This is evidenced by construction of the answer, which opens with the "but": But we have not got a long term relation yet.

The main function - through the image of a temporary restriction (have not got a long term relation yet) to show the boundedness of the common space.

Emotional background: oscillation.

Movement from the space "We" into the space "I": I'm not ready ... The negative form of the predicate marks the further movement. The main type of determination is time: the man contrasts the present time (I am not ready) to the cognitive program of the future made by woman.

Values: readiness to expand the common space.

Scale: the decision taken by yourself is evaluated as "good"; under pressure it is evaluated as "bad".

The leading channel of perception: identification the confrontation "I" and "we".

The goal is to defend your own opinion and personal space.

Intensifiers:

Lexical: (not ready) reduces the value of duration to the minimum.

Syntactical: Both statements within one answer contain elements of negation (not... a long, not ready).

(Masha) I have to know the result this relationship leads to!

The main function is to persuade the interlocutor (I have to) change his mind and give another answer.

Emotional background: indignation. She heard the answer, but she is not ready to accept it, so she tries to force the man to leave the space "now" and to join the "future" space and impose obligations.

The main type of determination is time: connect the vector of the present (have to know (now) with the future vector (the result this relationship leads to!).

Values: confidence in the prospects for future events.
Scale: the willingness of a partner to marriage and children is evaluated as "good", unwillingness is evaluated as "bad".

Leading channel of perception: emotional. Complex arguments are replaced by emotionally rich exclamation.

Intensifiers:

Lexical: modal suggestion (have to know), the expression (the result this relationship leads to!), pronoun (this).

Syntactical: an exclamation form of the utterance.

Despite perseverance and emotional filling, women quickly cool down and change their mind: (a week after the quarrel) (Masha) There is no need for the offer. I thought, I was wrong, and children could be given birth after 30 .

The main function is to persuade the interlocutor to reconcile.

Emotional background: regret.

The leading type of temporal determination is the connection of the past vector with the vector of the present and the future (I thought I was wrong, there is no need, could be given ...after) taking into account the desires and expectations of the partner.

Values: maintaining relationships and personal plans.

Scale: the restoration of relations and the preservation of the prospects for marriage and having children are evaluated as "good", parting and loneliness are evaluated as "bad".

The leading channel of perception: rational. Statements with elements of introspection I thought I was wrong). The modality of necessity replaces the modality of the obligation (no need) and assumptions (could).

Intensifiers:

Syntactical: impersonal construction (There is no need).

Emphasis on oneself (I thought, I was wrong).

\subsection{Forecasting}

Women draw conclusions on behalf of men about themselves:

(Masha) I can't be the first to call. He will decide that he is interesting for me. And he should not know.

(Masha) It is necessary to prove that I have been busy with something, or he will think that I have been sitting and waiting, and he will consider me an unrealized woman.

(Masha) He is not ringing anymore. Now he will think that I'm a ridiculous hen who does not know where her phone is.

The main function is to form a positive opinion about yourself (he should not know, will consider me unrealized, he will think that I'm ridiculous).

Emotional background: fear of making a bad impression.

The leading type of determination is time: projecting the role of a partner and his reactions (he will decide, he will think, he will consider me).

Values: good opinion of a partner. 
Scale: a good impression produced on a man is evaluated as "positive" for the future relationship development, a bad impression is evaluated as "negative" for the future relationship development.

The leading channel of perception: rational in relation to the man and emotional in relation to herself (to be interesting for somebody, unrealized, ridiculous hen).

Different types of modality explicate the probability models of reality and role behaviour (I can't be the first to phone, he should not know, it is necessary to prove).

A woman tries to convert her wish into the reality in order to improve her own position the man pays attention for (It is necessary to prove that I have been busy with something, or he will think that I have been sitting and waiting, and he will consider me an unrealized woman. He will decide, that he is interesting for me. And he should not know).

Intensifiers:

Syntactic: complex reasoning (or, and, that, who, where).

Lexical: emotional nomination (ridiculous hen).

\section{Conclusion}

\subsection{Results}

In the analysis of female speech discourse, it was discovered that the core spheres of male interest in female perception are women themselves and alcohol.

The following male behaviour standards connected with women were identified. The man pays the most attention to the woman's shape and beauty. Then we must mention that man wants innocence and passion to be combined. For the long-term relations, it is important woman to be intelligent and active in profession and in social life.

Female perception analysis shows that women often try to influence (with a little success) on the picture of the world and on the values and orientations of men, trying to match them with their own.

Spheres of women's interest are work, recreation, personal life, plans for the future, marriage, family, children, friends.

It was observed that women, in comparison with men, are stronger been influenced and have greater dependence on social and cultural stereotypes and roles.

Male receptive standards about appearance and behaviour give birth to a great need for women to match with it (to look attractive, to get married, to have children, to be active at work, to have many interests and friends).

On the language level, this is evidenced by the multiple inclusions of the modal components of need and necessity in the discourse of the utterance (is no need, could be, should not know, would be, I have to know, you would remove).

\subsection{Conclusion}

Dominant psychoemotional background of female verbal behaviour - a desire or dream. Speech reproduction of the male receptive stereotypes has a detailed character; it is included into the female speech in the form of complex sentences. If the male standard does not suit the female standard, the woman fills with emotions, and her speech fills with exclamations, emotional words with affective character. Speech behaviour of a woman reflects the orientation toward the value of the partner's world and reflects the desire for the best future in an idealistic way.

\section{References}

1. K. Sedov, Genre nature of the discursive thinking of the linguistic personality (Genres, Saratov, 1999)

2. E. Goroshko, A. Kirilina Gender Studies J., Kharkov 2, 234-241 (1999)

3. A. Kirilina, Woman in Russian Society J. 2, 21-27 (1998)

4. M. Gorodnikova, The Gender Factor and the Distribution of Social Roles in Modern Society, The Gender Factor in Language and Communication, Ivanovo, 23-27 (1999)

5. I. Sternin, Problem of the description of gender communicative behaviour (Gender: language, culture, communication, Moscow, 2003)

6. V. Voichenko Vestnik of Volgograd State University J. 1 (9), 64-65 (2009)

7. Yu. Karaulov, Russian language and language personality, (Science, Moscow, 1987).

8. V. Maslova, Expressiveness Parameters of the text, Human factor in the language. Language mechanisms of expressiveness. Moscow, 179-204 (1991)

9. V. Shakhovsky Categorization of emotions in the lexical and semantic system of language. Voronezh 85, (1987).

10. R. Fiehler, Kommunikation und Emotion, (Walter de Gruyter, Berlin; New York, 1990).

11. V. Dementiev, Communicative genre: speech genres as a means of formalizing social interaction, Genres of speech, Saratov 3, 18-40 (2002)

12. A. Baranov, Two structural components in cogniotype and its genre peculiarities (Genres, Saratov, 1999)

13. A. Kirilina, Some results of gender studies in Russian linguistics (Gender: language, culture, communication, Moscow, (2000)

14. M. Bakhtin, The problem of speech genres, Russian vocabularies (Moscow, 1996) 